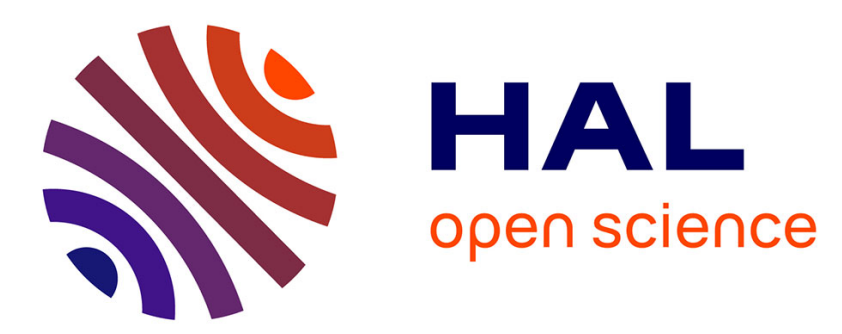

\title{
Local turn-boundedness: a curvature control for a good digitization
}

\author{
Etienne Le Quentrec, Loïc Mazo, Etienne Baudrier, Mohamed Tajine
}

\section{To cite this version:}

Etienne Le Quentrec, Loïc Mazo, Etienne Baudrier, Mohamed Tajine. Local turn-boundedness: a curvature control for a good digitization. 21st IAPR International Conference on Discrete Geometry for Computer Imagery, Mar 2019, Paris, France. hal-01898138

\section{HAL Id: hal-01898138 \\ https://hal.science/hal-01898138}

Submitted on 18 Oct 2018

HAL is a multi-disciplinary open access archive for the deposit and dissemination of scientific research documents, whether they are published or not. The documents may come from teaching and research institutions in France or abroad, or from public or private research centers.
L'archive ouverte pluridisciplinaire HAL, est destinée au dépôt et à la diffusion de documents scientifiques de niveau recherche, publiés ou non, émanant des établissements d'enseignement et de recherche français ou étrangers, des laboratoires publics ou privés. 


\title{
Local turn-boundedness: a curvature control for a good digitization
}

\author{
Étienne Le Quentrec, Loïc Mazo, Étienne Baudrier and Mohamed Tajine \\ ICube-UMR 7357, 300 Bd Sébastien Brant - CS 10413 \\ 67412 Illkirch Cedex France \\ elequentrec@unistra.fr
}

\begin{abstract}
This paper focuses on the classical problem of the control of information loss during the digitization step. The properties proposed in the literature rely on smoothness hypotheses that are not verified by the curves including angular points. The notion of turn introduced by Milnor in the article On the Total Curvature of Knots generalizes the notion of integral curvature to continuous curves. Thanks to the turn, we are able to define the locally turn-boundedness. This promising property of curves do not require smoothness hypotheses and shares several properties with the par $(r)$-regularity, in particular well-composed digitizations. Besides, the locally turn-boundedness enables to constraint spatially the continuous curve in function of its digitization.
\end{abstract}

\section{Introduction}

The loss of information caused by a digitization process is inevitable. Therefore a fundamental point concerns the control of this information loss. Indeed, the border of a compact connected shape $S$ can be arbitrarily far from the digitization of $S$ or can oscillate around this latter border. Therefore, hypotheses on the border of the shape $S$, which is a Jordan curve noted by $\mathcal{C}$, are needed. One of the most used hypothesis, called par(r)-regularity, has been introduced by Pavlidis in 7. It demands that any point $c \in \mathcal{C}$ has an interior osculating disk entirely included in the interior of $\mathcal{C}$ except for the point $c$ and an exterior osculating disk entirely included in the exterior of $\mathcal{C}$ except for the point $c$. It has been used to prove some preserving topology properties 2, 5, 7, or to control the behavior of the projection from the digitized curve to the continuous curve $\mathcal{C}$ [3, 4]. However, the notion can be hard to manipulate in geometry and most of the authors using this notion add the assumption that the curve $\mathcal{C}$ is of class $C^{2}$. The par $(r)$-regularity encompasses two ideas: the border of the shape has a bounded curvature from above and the shape has a positive minimal thickness. In this article, relaxing the assumption of a bounded curvature, we develop a new notion called local turn-boundedness that is defined on continuous curves, including polygons. The local turn-boundedness relies on the notion of turn adapted to regular curves and polygons, firstly introduced by Milnor 6 to study the geometry of knots. The main properties and definitions around the 
notion of turn are recalled in Section 2. The local turn-boundedness involves the Euclidean distance between any two points of a curve and the turn of this curve between these two points. The properties of locally turn-bounded curves are given and illustrated in Section 3 .

\section{Turn of a simple curve}

The definitions and properties given in this section are detailed in 1]. As presented in Proposition 2, the turn extends to continuous curves the notion of integral curvature already defined for regular curves.

\section{Terminology and notations}

- Let $c \in \mathbb{R}^{2}$ and $r \geq 0, B(c, r)$ is the open disk of center $c$ and radius $r$.

- A parametrized curve is a continuous application from an interval $[a, b]$ of $\mathbb{R}$ $(a<b)$ to $\mathbb{R}^{2}$. It is simple if it is injective on $[a, b)$ and closed if $\gamma(b)=\gamma(a)$. A (geometric) curve is the image of a parametrized curve. A Jordan curve is a simple closed curve.

- A polygonal line with vertices $x_{0}, \ldots, x_{N}$ is noted $\left[x_{0} x_{1} \ldots x_{N}\right]$ (if $x_{N}=x_{0}$, the polygonal line is a polygon).

- Let $N$ a positive integer and $x_{0}, x_{1}, \ldots, x_{N}$ points of $\mathbb{R}^{2}$. The polygonal line $\mathrm{PL}=\left[x_{0} x_{1} \ldots x_{N}\right]$ can be considered as the image of the parametrized curve $p l:[0, N] \mapsto \mathbb{R}^{2}$ such that $p l(t)=x_{\lfloor t\rfloor}(t-\lfloor t\rfloor)+(1-t+\lfloor t\rfloor) x_{\lfloor t\rfloor+1}$ where for $r \in \mathbb{R},\lfloor r\rfloor$ in the integer part of the real $r$. In other words, for any integer $i$ between 0 and $N$, if $t \in[i, i+1)$, then $p l(t)=(t-i) x_{i}+(1-t+i) x_{i+1}$, and thus $p l([i, i+1])$ is the segment $\left[x_{i}, x_{i+1}\right]$ of $\mathbb{R}^{2}$. A polygonal line is simple if it is simple for the previous parametrization and thus a simple polygon is a Jordan curve.

- Given a curve $\mathcal{C}$ and two points $a, b$ on $\mathcal{C}(a \neq b)$, we write $\mathcal{C}_{a}^{b}$ for the arc ending at $a$ and $b$ if $\mathcal{C}$ is not closed and $\mathcal{C}_{a}^{b}$ and $\mathcal{C}_{b}^{a}$ for the two arcs of $\mathcal{C}$ ending at $a$ and $b$ if $\mathcal{C}$ is closed.

- The angle between two vectors $\boldsymbol{u}$ and $\boldsymbol{v}$ is noted $(\boldsymbol{u}, \boldsymbol{v})((\boldsymbol{u}, \boldsymbol{v}) \in \mathbb{R} / 2 \pi \mathbb{Z})$. The geometric angle between two vectors $\boldsymbol{u}$ and $\boldsymbol{v}$, noted $(\hat{\boldsymbol{u}, \boldsymbol{v}})$, or two directed straight lines oriented by $\boldsymbol{u}$ and $\boldsymbol{v}$, is the absolute value of the reference angle taken in $(-\pi, \pi]$ between the two vectors. Thus, $(\hat{\boldsymbol{u}, \boldsymbol{v}}) \in[0, \pi)$.

\section{Definition 1 (Turn).}

- The turn $\kappa(L)$ of a polygonal line $L=\left[x_{i}\right]_{i=0}^{N}$ is defined by:

$$
\kappa(L):=\sum_{i=1}^{N-1}\left(x_{i-1} \widehat{x_{i}, x_{i}} x_{i+1}\right) .
$$

- The turn $\kappa(P)$ of a polygon $P=\left[x_{i}\right]_{i=0}^{N}$ (where $x_{N}=x_{0}$ ) is defined by:

$$
\kappa(P):=\sum_{i=1}^{N}\left(x_{i-1} \widehat{x_{i}, x_{i}} x_{i+1}\right) .
$$


- The turn $\kappa(\mathcal{C})$ of a simple curve $\mathcal{C}$ is the upper bound of the turn of its inscribed polygonal lines

- The turn $\kappa(\mathcal{C})$ of a Jordan curve $\mathcal{C}$ is the upper bound of the turn of its inscribed polygons.

It should be noticed that the turn does not depend of the orientation of the curve. Indeed, it is well-known that $(\boldsymbol{u}, \boldsymbol{v})=(-\boldsymbol{u},-\boldsymbol{v})=-(-\boldsymbol{v},-\boldsymbol{u})$. Thus $\kappa\left(\left[x_{i}\right]_{i=0}^{N}\right)=\kappa\left(\left[x_{i}\right]_{i=N}^{0}\right)$.

Furthermore, since the turn of a polygon is equal the upper bound of the turn of the polygonal lines inscribed in it (cf Corollary p. 119 [1]), the turn of the polygon seen as a closed curve is equal to the turn of the polygon. Hence the notation $\kappa$ is well defined.



Figure 1: The turn of the polygon is the sum of the green angles.

In the same way that we estimate the length of a curve, the following proposition make it possible to calculate the turn thanks to multiscale samplings. Given a curve $\mathcal{C}$, we denote by $\mathcal{L}(\mathcal{C})$ the length of $\mathcal{C}$.

Proposition 1 (Convergence of the length and turn of a sequence of polygonal lines $[\mathbf{1}], \mathbf{p} . \mathbf{2 3}, \mathbf{3 0}, \mathbf{1 2 1}, \mathbf{1 2 2})$. Let $\mathcal{C}$ be a simple curve and $\left(L_{m}\right)_{m \in \mathbb{N}}$ a sequence of polygonal lines inscribed in $\mathcal{C}$ and with same endpoints as $\mathcal{C}$. If $\lim _{m \rightarrow+\infty} \lambda_{m}=0$, where $\lambda_{m}$ is the maximal Euclidean distance between two consecutive vertices of $L_{m}$, then

$$
\lim _{m \rightarrow+\infty} \mathcal{L}\left(L_{m}\right)=\mathcal{L}(\mathcal{C})
$$

and

$$
\lim _{m \rightarrow+\infty} \kappa\left(L_{m}\right)=\kappa(\mathcal{C}) .
$$

Moreover, if $\kappa(\mathcal{C})$ is finite, then $\mathcal{L}(\mathcal{C})$ is also finite, that is $\mathcal{C}$ is rectifiable.

In Prop. 1 if we assume that the sequence $\left(L_{m}\right)$ is increasing $\left(L_{m}\right.$ is inscribed in $\left.L_{m+1}\right)$, then the sequences $\left(\mathcal{L}\left(L_{m}\right)\right)$ and $\left(\kappa\left(L_{m}\right)\right)$ are both increasing $([1$, Lemma 5.1.1]). 
Proposition 2 (Turn for regular curves $[\mathbf{1}]$, p. 133). Let $\gamma:[0, \ell] \rightarrow \mathbb{R}^{2}$ be a parametrization by arc length of a simple curve $\mathcal{C}$. Assume that $\gamma$ is of class $C^{2}$ and denote by $k(s)$ the curvature at the point $\gamma(s)$. Then,

$$
\kappa(\gamma)=\int_{0}^{\ell} k(s) \mathrm{d} s
$$

For regular curves, therefore, the turn corresponds to the integral of the curvature (with respect to an arc-length parametrization).

The following theorem gives a lower bound of the turn for closed curves.

Theorem 1 (Fenchel's Theorem, [1] theorem 5.1.5 p.125). For any Jordan curve $\mathcal{C}, \kappa(\mathcal{C}) \geq 2 \pi$. Moreover $\kappa(\mathcal{C})=2 \pi$ if and only if the interior of $\mathcal{C}$ is convex.

Proposition 3. Let $\mathcal{C}$ be a simple curve from a to $b$ and $\gamma$ be its parametrization. Let $\mathcal{C}_{a}^{x_{2}}$ and $\mathcal{C}_{x_{1}}^{b}$ be two arcs of $\mathcal{C}$ that overlap with $a<x_{1}<x_{2}<b$. Then,

$$
\kappa(\mathcal{C}) \leq \kappa\left(\mathcal{C}_{a}^{x_{2}}\right)+\kappa\left(\mathcal{C}_{x_{1}}^{b}\right)
$$

Proof. Let $\left(L_{m}\right)$ be a sequence of polygonal lines $\left[l_{m, i}\right]_{i=0}^{N_{m}}$ inscribed in $\mathcal{C}$ such that $l_{m, 0}=a, l_{m, N_{m}}=b$ and :

$$
\left\|l_{m, i+1}-l_{m, i}\right\|_{2} \leq \frac{1}{m}
$$

Let $m>2 /\left(x_{2}-x_{1}\right)$ and denote by $l_{m, i_{2}}$ the largest $l_{m, i}$ such that $l_{m, i}$ lies in $\mathcal{C}_{a}^{x_{2}}$ and $l_{m, i_{1}}$ the smallest $l_{m, i}$ that lies in $\mathcal{C}_{x_{1}}^{b}$. Then,

$$
l_{m, i_{1}}<l_{m, i_{2}}
$$

Therefore, from Definition 1] we derive that

$$
\kappa\left(\left[l_{m}\right]_{i=0}^{N_{m}}\right) \leq \kappa\left(\left[l_{m}\right]_{i=0}^{i_{2}}\right)+\kappa\left(\left[l_{m}\right]_{i=i_{1}}^{N_{m}}\right) .
$$

Then, by Proposition 1

$$
\kappa(\mathcal{C}) \leq \kappa\left(\mathcal{C}_{a}^{x_{2}}\right)+\kappa\left(\mathcal{C}_{x_{1}}^{b}\right)
$$

Without the hypothesis of an overlapping non reduced to a singleton, Proposition 3 is false in presence of angular points as it is illustrated in Figure 2 .

Remark 1. The turn is stable under homothetic maps. Indeed, obviously, the turn is invariant by any conformal map, in particular by the homotheties. 


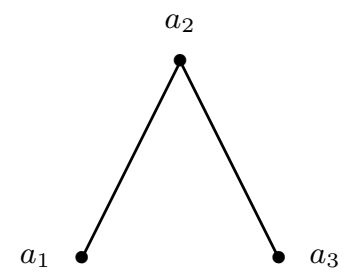

Figure 2: Counterexample: the turn of the $\operatorname{arcs} \mathcal{C}_{a_{1}}^{a_{2}}$ and $\mathcal{C}_{a_{2}}^{a_{3}}$ are zero but the turn of the $\operatorname{arc} \mathcal{C}_{a_{1}}^{a_{3}}$ is nonzero. This is why the arcs are required to be overlapping in Proposition 3

\section{Locally turn-bounded curves}

Definition 2. Let $\theta \geq 0$. The $\theta$-turn step $\sigma(\theta)$ of a Jordan curve $\mathcal{C}$ is the infimum of the (Euclidean) distances between two points a and $b$ in $\mathcal{C}$ such that the turns of $\mathcal{C}$ between $a$ and $b$ are both greater than $\theta:$

$$
\sigma(\theta)=\inf \left\{\|a-b\|_{2} \mid \kappa\left(\mathcal{C}_{a}^{b}\right)>\theta \wedge \kappa\left(\mathcal{C}_{b}^{a}\right)>\theta\right\} .
$$

Let $\theta \geq 0, \delta \geq 0$. A Jordan curve $\mathcal{C}$ is locally turn-bounded with parameters $(\theta, \delta)$ if $\delta \leq \sigma(\theta)$.

The Figure 3 illustrates the definition of the local turn with different curves.

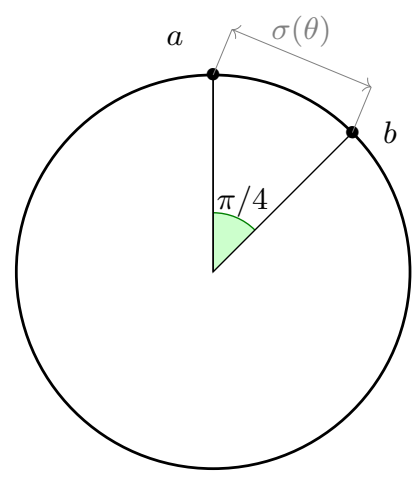

$\theta=\pi / 4$

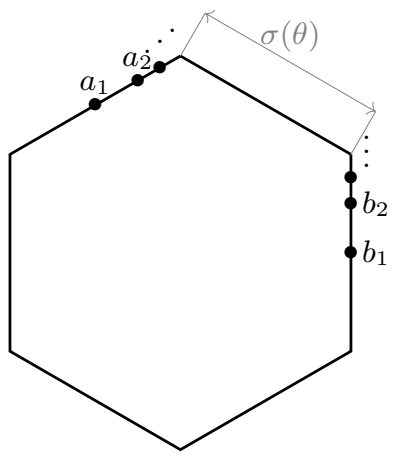

$\theta=\pi / 3$

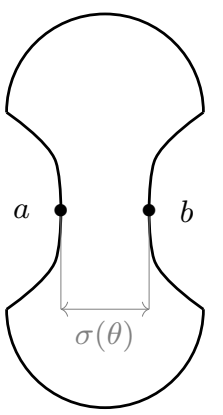

$\theta=\pi / 2$

Figure 3: For the chosen value of $\theta$, the corresponding $\theta$-turn-step $\sigma(\theta)$.

On a locally turn-bounded curve with parameters $(\theta, \delta)$ the smallest turn of the curve $\mathcal{C}$ between two points of $\mathcal{C} a$ and $b$ such that $\|b-a\|_{2}<\delta$ is less than or equal to $\theta$. 
The turn step function $\theta \mapsto \sigma(\theta)$ is increasing. Indeed, the value of the $\theta$-turn step is an infimum of a set that decreases (for the inclusion order) in function of $\theta$. If the turn of $\mathcal{C}$ is finite, it exists a value $\theta_{\max }$ above which this set is empty and the $\theta_{\max }$-turn step is then infinite.

\section{Examples}

- The 0-turn step of a Jordan curve is 0.

- The $\pi$-turn step of a convex Jordan curve is $+\infty$ (see Theorem 1).

- The $\theta$-turn step of a circle with radius $r$ is $2 r \sin (\theta / 2)$ if $\theta \leq \pi$ and is infinite for $\theta>\pi$.

- The $\theta$-turn step of a polygon having vertices with interior angles strictly less than $\pi-\theta$ is zero. Indeed, the turn of an arc joining two points arbitrary close, located before and after such a vertex is greater than $\theta$.

- The turn between two points on a polygonal curve is a finite sum of geometric angles, then the turn step function is a step function.

Remark 2. Local turn-boundedness is scale invariant: let $\mathcal{C}$ be a locally turnbounded Jordan curve with parameters $(\theta, \delta)$. Then, the curve $k \mathcal{C}, k>0$, is locally turn-bounded with parameters $(\theta, k \delta)$. It is a direct consequence of Remark 1 .

The next proposition makes it possible to localize a locally turn-bounded curve from a sufficiently tight sampling. Figure 4 illustrates the proposition.

Proposition 4. Let $\mathcal{C}$ be a simple curve locally turn-bounded with parameters $(\theta \in(0, \pi), \delta)$. Let $a<b$ be two points on $\mathcal{C}$ such that $\|a-b\|_{2}<\delta$. Then, one of the arc of $\mathcal{C}$ between $a$ and $b$ is included in the union of the two truncated closed disks where the line segment $[a, b]$ is seen from an angle greater than or equal to $\pi-\theta$.

Proof. Since the Euclidean distance between $a$ and $b$ is less than the $\theta$-turn step, the turn of one of the arc of $\mathcal{C}$ between $a$ and $b$ is less than or equal to $\theta$. Denote by $\mathcal{C}_{0}$ this arc. Let $c$ be a point on $\mathcal{C}_{0}$. By definition, the turn of the polygonal line $[a, c, b]$ is less than or equal to the turn of $\mathcal{C}_{0}$ Then the geometric angle $(a-\widehat{c, b}-c)$ is greater than or equal to $\pi-\theta$. We conclude the proof by invoking the inscribed angle theorem.

Locally turn-bounded curves for angles $\theta \leq \pi / 2$ are locally connected subsets of the Euclidean plane.

Proposition 5. Let $\mathcal{C}$ be a locally turn-bounded Jordan curve with parameters $(\theta \in(0, \pi / 2], \delta)$ and $a \in \mathcal{C}$. Then the intersection of $\mathcal{C}$ with the open disk $D(a, \epsilon)$ is connected whenever $\epsilon \leq \delta$.

Proof. Let $a \in \mathcal{C}$. Let $b \in \mathcal{C} \cap D(a, \epsilon)$. Then, by the very definition of $\delta$, the turn of one of the $\operatorname{arc}$ of $\mathcal{C}$ between $a$ and $b$ is less than or equal to $\theta$. So, from Prop. 4 and for $\theta \leq \pi / 2$, this arc is included in the disk with diameter $[a, b]$ which is itself included in $D(a, \epsilon)$. Hence, $\mathcal{C} \cap D(a, \epsilon)$ is path-connected. 


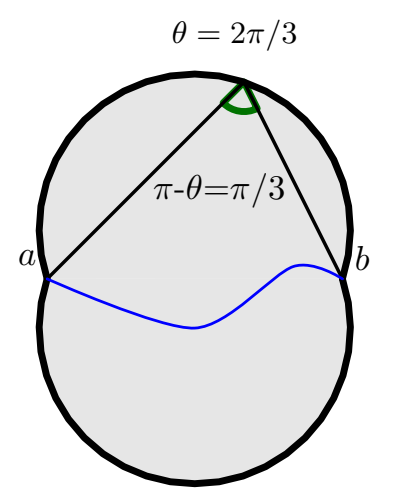

$\theta=\pi / 2$

$\theta=\pi / 3$

Figure 4: Illustration of Prop. 4 for three values of the parameter $\theta: \pi / 3, \pi / 2$, $2 \pi / 3$. Given two points $a, b \in \mathcal{C}$ such that $\|a-b\|_{2}<\sigma(\theta)$, then one of the arc of $\mathcal{C}$ between $a$ and $b$ belongs to the grey area.

For $\pi / 2<\theta<\pi$, in particular for polygons with acute angles, Proposition 5 does not hold (the intersection of the curve with a ball near an acute angle may have two connected components). Nevertheless, a weak version of the proposition could be stated: the intersection of the disk $D(a, \delta)$ with the dilation of $\mathcal{C}$ by the structuring element $B(0,(1 / \sin \theta-1) \delta)$ is connected.

Proposition 6. A locally turn-bounded curve with parameters $(\theta \in(0, \pi / 2], \delta)$ has a finite turn and is thus rectifiable.

Proof. Let $\mathcal{C}$ be a locally turn-bounded curve with parameters $(\theta, \delta)$. The open balls $B(a, \delta / 2), a \in \mathcal{C}$, cover the compact set $\mathcal{C}$. Then, there exists a finite subset of $\mathcal{C},\left\{a_{0}, \ldots, a_{m}\right\}$ such that $\bigcup_{i=0}^{m} B\left(a_{i}, \delta / 2\right)$ covers $\mathcal{C}$. By Proposition 5 , for each $i, \mathcal{C} \cap B\left(a_{i}, \delta / 2\right)$ is an $\operatorname{arc}$ of $\mathcal{C}$.

Since the balls are open and thus overlaps, by Proposition $3 \kappa(\mathcal{C}) \leq \sum_{i=0}^{m} \kappa(\mathcal{C} \cap$ $\left.B\left(a_{i}, \delta / 2\right)\right)$. Besides, by hypothesis, $\kappa(\mathcal{C} \cap B(a, \delta / 2) \leq \theta$. Therefore, $\kappa(\mathcal{C}) \leq$ $(m+1) \theta$.

The next technical lemma is used in the proofs of Proposition 8 and Proposition 7

Lemma 1. Let $\mathcal{C}$ be a curve with endpoints $a, b$ such that the line segment $[a, b]$ does not intersect the curve $\mathcal{C}$. Let $P$ be a polygonal line from a to $b$ lying in the interior of the Jordan curve $\mathcal{C} \cup[a, b]$ and such that $P \cup[a, b]$ is convex. Then $\kappa(C) \geq \kappa(P)$.

Proof. Let $c$ be any point in $(a, b)$ and $Q=\left[a q_{1} \ldots q_{m} b\right]$ the polygonal line obtained by projecting $P=\left[a p_{1} \ldots p_{m} b\right]$ on $\mathcal{C}$ from $c$ (see Fig. 5). Then, $\kappa(C) \geq$ $\kappa(Q)$ by definition of $\kappa(\mathcal{C})$. Besides, $\kappa(Q \cup[b, a]) \geq \kappa(P \cup[b, a])$, for $P \cup[b, a]$ is convex, and $\kappa\left(\left[q_{m} b a q_{1}\right]\right) \leq \kappa\left(\left[p_{m} b a p_{1}\right]\right)$ by construction of $Q$. Since $\kappa(Q \cup[b, a])=$ $\kappa(Q)+\kappa\left(\left[q_{m} b a q_{1}\right]\right)$ and $\kappa(P \cup[b, a])=\kappa(P)+\kappa\left(\left[p_{m} b a p_{1}\right]\right)$, the result holds. 


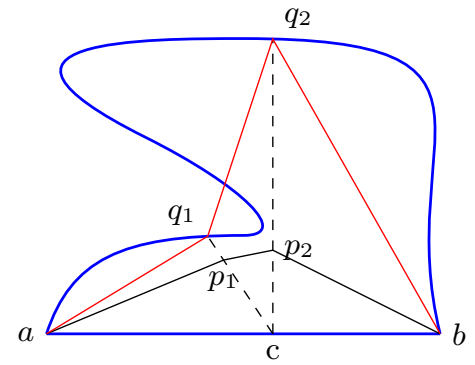

Figure 5: Thick, blue: the curve $\mathcal{C}$ and the line segment $[a, b]$. Black: the polygonal line $P=\left[a p_{1} p_{2} b\right]$. Black, dashed: the projection of $p_{1}$ and $p_{2}$ on $\mathcal{C}$ yields the points $q_{1}$ and $q_{2}$. Red: the polygonal line $Q=\left[a q_{1} q_{2} b\right]$.

The information on the local turn makes it possible to localize the continuous curve from its digitization as illustrated by Fig. 6 .

Proposition 7 (Hausdorff distance between the curve and a pixel). Assuming an $n$-regular tiling of the plane with edge length $h(n \in\{3,4,6\})$, let $\mathcal{C}$ be a locally turn-bounded Jordan curve with parameters $(\theta<2 \pi / n, \delta>$ $h \sqrt{n-2})$. Let $T$ be a tile crossed by $\mathcal{C}$ and $a, b$ be respectively the infimum and the supremum of $\mathcal{C} \cap T$ (C is ordered by some parametrization). Then, the Hausdorff distance between $T$ and one of the segments of $\mathcal{C}$ bounded by $a$ and $b$ is less than $h(1-\cos (\theta)) / 2 \sin (\theta)$.

Proof. Let $\mathcal{C}_{1}, C_{2}$ be the arcs of $\mathcal{C}$ bounded by $a$ and $b$. We assume $\kappa\left(\mathcal{C}_{1}\right) \leq \kappa\left(\mathcal{C}_{2}\right)$ (then, $\kappa\left(\mathcal{C}_{2}\right) \geq \pi>\theta$ ). As the diameter of $T$ is $\sqrt{n-2} h$, by the hypothesis $\delta \geq \sqrt{n-2} h, \kappa\left(\mathcal{C}_{1}\right) \leq \theta$. If the arc $\mathcal{C}_{1}$ leaves $T$, it intersects the border of $T$ on a point $c$. By the definition of $a$ and $b$ there exists $d \neq c$ such that $d$ is on the border of $T$. The point $d$ belongs to the same edge as $c$. Indeed, if it wasn't the case, there would be a polygonal line included in the border of $T$ containing at least one vertex and separating an $\operatorname{arc}$ segment of $\mathcal{C}_{1}$ from the line segment $[c, d]$. Then, by Lemma 1 the turn of $\mathcal{C}_{1}$ would be greater than or equal to $2 \pi / n>\theta$. Hence, $c$ and $d$ belongs to the same edge. Therefore, by the proposition 4 , we derive that $\mathcal{C}_{1}$ lies in the union of $T$ with the interior of $n$ truncated circles whose Hausdorff distance to $T$ is $h(1-\sin \theta) / 2 \cos \theta$.

The next proposition makes a link between the well-composedness of the digitized object and a local turn-boundedness hypothesis. Let us first define the Gauss digitization and the well-composedness. Let $h>0$ be a sampling grid step, the Gauss digitization of a shape $K$ is defined as $K \cap(h \mathbb{Z})^{2}$. By abuse of language, given a Jordan curve $\mathcal{C}$ which is the border of the compact shape $K$, we define its Gauss digitization - we write $\operatorname{Dig}_{h}(\mathcal{C})$ - as the border of the union of the squares $p \oplus[-h / 2, h / 2] \times[-h / 2, h / 2]$ where $\oplus$ denotes the Minkowski sum and $p \in K \cap(h \mathbb{Z})^{2}$. The Gauss digitization of $\mathcal{C}$ is well-composed if it is a Jordan curve. 

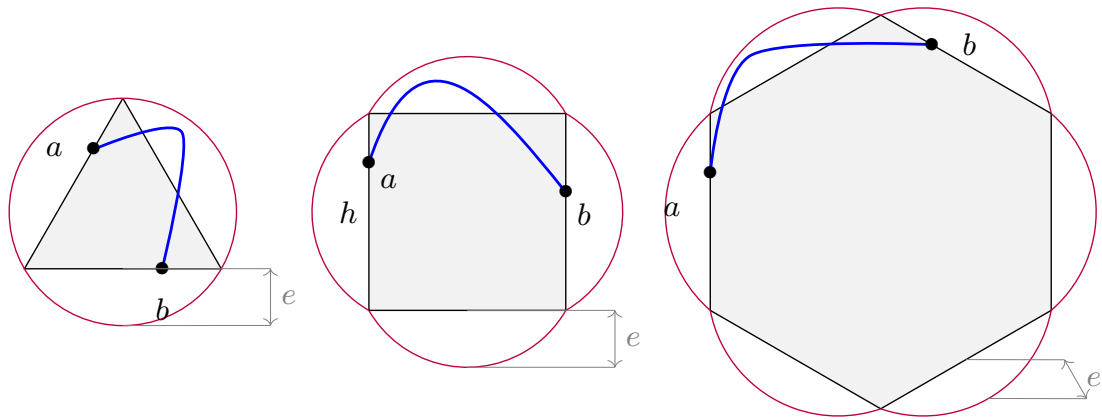

$$
\begin{aligned}
& \theta=\frac{\pi}{3}, \sigma>h \\
& e=h(\sqrt{3} / 6)
\end{aligned}
$$

$$
\begin{gathered}
\theta=\frac{\pi}{4}, \sigma>2 h \\
e=h(\sqrt{2}-1) / 2
\end{gathered}
$$

Figure 6: Gray: a tilel $T$ with edge length $h$. Blue, thick: a locally turn-bounded curve arc passing through $T$. Red: the union of $n$ circle arcs from which the edges of $T$ are viewed from an angle $2 \pi-\theta$. The Hausdorff distance between the circle arcs and the pixel $T$ is $e$.

Proposition 8. Let $\mathcal{C}$ be a locally turn-bounded Jordan curve with parameters $(\theta \in(0, \pi / 2], \delta)$ where $\delta$ is less than the diameter of $\mathcal{C}$. Then, the Gauss digitization of $\mathcal{C}$ for a grid step $h<\delta / \sqrt{2}$ is almost surely a disjoint union of Jordan curves (the digitization of the shape is well-composed).

Proof. The proof is made by contradiction. So, let $a$ be a double point on $\operatorname{Dig}_{h}(\mathcal{C})$ traveled counterclockwise. Since the interior points of $(h \mathbb{Z})^{2}$ are on the left of the discrete curve, there is only one configuration modulo rotations and symmetries depicted in Fig 7. Furthermore, we can assume almost surely $\mathcal{C} \cap(h \mathbb{Z})^{2}=\emptyset$. Indeed, the set of translations for which a given point $x \in(h \mathbb{Z})^{2}$ lies on the translation of $\mathcal{C}$ is $\{x-p \mid p \in \mathcal{C}\}$. In other words, it is a translation of $-\mathcal{C}$ (in the translation parameter space). Then, since $\mathcal{C}$ is rectifiable, this set is onedimensional. Therefore, the set $S$ of translations for which there exists a point of $(h \mathbb{Z})^{2}$ on the translation of $\mathcal{C}$ is a countable union of one-dimensional sets. Thus, the Lebesgue measure of $S$ is zero.

With the notation of the figure, we claim that on each edge of the square $T=\left[I_{1}, E_{1}, I_{2}, E_{2}, I_{1}\right]$ lies a point of the curve $\mathcal{C}$. Indeed, each edge links an interior point and an exterior point. Furthermore, thanks to the assumptions that $\mathcal{C} \cap(h \mathbb{Z})^{2}=\emptyset$, these four points actually lie on the open edges of $T$. Let $b, d$ be respectively the infimum and the supremum of $\mathcal{C} \cap T$ and $\left[c c_{0} c_{1} c_{2} c_{3} d\right]$ be the inscribed polygon formed by these six points $(\mathcal{C}$ is ordered by some parametrization). We denote by $\mathcal{C}_{b}^{d}$ the oriented arc of $\mathcal{C}$ from $b$ to $d$. On the one hand, the $\operatorname{arc} \mathcal{C}_{b}^{d}$ contains the four points $c_{0}, c_{1}, c_{2}$ and $c_{3}$. Thus, whatever the geometric configuration ( $\left[c_{0} c_{1} c_{2} c_{3}\right]$ simple or not), the turn of $\mathcal{C}_{b}^{d}$ is greater than $\pi / 2$. Nevertheless, since $\delta>h \sqrt{2}$, the points $b, c_{0}, c_{1}, c_{2}, c_{3}, d$ are included in the ball 


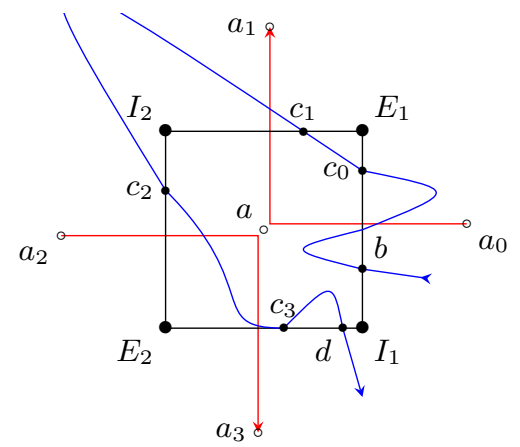

Figure 7: Blue: the curve $\mathcal{C}$. Red: the line segments $\left[a_{0} a\right],\left[a a_{1}\right],\left[a_{2} a\right],\left[a a_{3}\right]$ are edges of $\operatorname{Dig}_{h}(\mathcal{C})$. Black: the points $E_{1}, E_{2}, I_{1}, I_{2}$ are in $(h \mathbb{Z})^{2}, E_{1}, E_{2}$ are exterior to $\mathcal{C}$ while $I_{1}, I_{2}$ are interior to $\mathcal{C}$.

$B(a, \delta)$. Thus, from Prop. 5 , the $\operatorname{arc} \mathcal{C}_{b}^{d}$ is included in the ball $B(a, \delta)$. On the other hand, since $\kappa\left(C_{b}^{d}\right) \geq \pi / 2$ and $\delta>h \sqrt{2}$, the turn of $\mathcal{C} \backslash \mathcal{C}_{b}^{d}$ is less than $\theta$ and a fortiori less than $\pi / 2$. Then, from Prop. 4. $\mathcal{C} \backslash \mathcal{C}_{b}^{d}$ is included in the disk with diameter $[b, d]$ which is itself included in the disk $B(a, \delta)$. Thereby, the whole curve $\mathcal{C}$ is included in $B(a, \delta)$ which contradicts the assumption on its diameter.

We began the proof of Prop 8 without any restriction on the localization of the curve (omitting "almost surely"). But there are many special cases to consider and it can not be given as part of a short article.

\section{Conclusion}

In this article, the notion of local turn, adapted to both regular curves and polygons having large enough interior angles, has been developed to have control on the curve without smoothness assumption. Indeed, it makes it possible to bound the domain where an arc of such a curve is lying, to guarantee (almost surely) the well-composedness of its Gauss digitization and to bound the Hausdorff distance between one pixel and an arc crossing it. The local turn needs to be related to others notions used in discrete geometry for similar purposes. To this end, we will prove in an upcoming article that a $\operatorname{par}(r)$-regular curve of class $\mathcal{C}^{1}$ is locally turn-bounded with parameters $(\theta, 2 r \sin (\theta / 2))$ where $\theta$ is any angle in $[0, \pi)$. Besides, we expect that these results will make it possible to correctly associate the vertices of a Gauss digitization to points on the continuous curve without smoothness assumption.

\section{References}

1. Alexandrov, Reshetnyak: General Theory of Irregular Curves 
2. Gross, A., Latecki, L.: Digitizations preserving topological and differential geometric properties

3. Lachaud, J.O.: Espaces non-euclidiens et analyse d'image : modèles déformables riemanniens et discrets, topologie et géométrie discrète

4. Lachaud, J.O., Thibert, B.: Properties of gauss digitized shapes and digital surfaces integration. Journal of Mathematical Imaging and Vision

5. Latecki, L.J., Conrad, C., Gross, A.: Preserving topology by a digitization process. Journal of Mathematical Imaging and Vision p. 131-159 (1998)

6. Milnor, J.W.: On the total curvature of knots (1949)

7. Pavlidis, T.: Algorithms for graphics and image processing (1982) 